Revue d'histoire de l'Amérique française

DEV RUE D.HISTOIRE DE L'AMÉRIQUE FRANÇAISE

\title{
Inventaire des meubles faisant partie de la communauté entre Samuel Champlain et Hélène Boullé
}

\section{Robert Le Blant}

Volume 18, numéro 4, mars 1965

URI : https://id.erudit.org/iderudit/302419ar

DOI : https://doi.org/10.7202/302419ar

Aller au sommaire du numéro

Éditeur(s)

Institut d'histoire de l'Amérique française

ISSN

0035-2357 (imprimé)

1492-1383 (numérique)

Découvrir la revue

Citer ce document

Le Blant, R. (1965). Inventaire des meubles faisant partie de la communauté entre Samuel Champlain et Hélène Boullé. Revue d'histoire de l'Amérique française, 18(4), 594-603. https://doi.org/10.7202/302419ar d'utilisation que vous pouvez consulter en ligne. 


\title{
DOCUMENTS INEDITS
}

\section{INVENTAIRE DES MEUBLES FAISANT PARTIE DE LA COMMUNAUTE ENTRE SAMUEL CHAMPLAIN ET HÉLENE BOULLE *}

\section{1 novembre 1636}

\begin{abstract}
L'an mil six cens trente six le vendredy apres midy vingt ungniesme jour de novembre et autres jours ensuivans, à la requeste de damoiselle Helayne Boullé vefve de feu messire Samuel de Champelain, vivant chevallier, seigneur dudit lieu et lieutenant pour le roy au gouvernement de la NouvelleFrance, demeurante à Paris rue d'Anjou, parroisse Saint-Jehan-en-Grève, tant en son nom à cause de la communaulté de biens d'entre ledit deffunt seigneur son mary et d'elle que comme donnataire dudict deffunt par son contrat de mariage, en la presence de damoiselle Marie Camaret, femme et procuratrice de maitre Jacques Hersant, controleur des gardes au bureau des traites fouraines et domanialles de la ville de La Rochelle, demeurant en ladite ville de La Rochelle, elle estant de present à Paris, logée en la Villeneufve-sur-Gravois, parroisse Saint-Eustache, fondée de procuration passée par devant Pierre Teuleron notaire et tabelion royal en ladite ville de la Rochelle en date du premier septembre dernier passé transcripte en fin des presentes et l'original à l'instant rendu à ladite damoiselle, icelle damoiselle habille à soy dire, nommer et porter heritiere du costé maternel dudit deffunt seigneur de Champelain son cousin germain et encores lesdits sieur et damoiselle Hersant creanciers de la succession dudit deffunt, aussy en la presence de noble homme Georges Lefebvre, conseiller du 1a Roy et antien substitud de Monsieur le procureur de Sa Majesté au Chatelet de Paris et à la conservation des droitz de qui il appartiendra et apres que le scellé apposé sur les biens demourez apres le decedz dudit deffunt seigneur de Champelain, a la requete de ladite damoiselle sa vefve par maitre Claude Le Vacher, commissaire et examinateur pour le Roy nostre sire au Chatelet de Paris, a esté par luy reconneu, levé et osté et (et) a esté par Guillaume Duchesne et Pierre Fieffé, notaires garde-nottes du Roy nostre sire au Chatelet de Paris faite description et inventaire de tous et chacuns les biens meubles et ustansilles d'hostel, habitz, linges, or et argent monnoyé et non monnoyé, lettres, tiltres, papiers et enseignemens demourez aprez le decedz dudit deffunt sieur de Champelain deceddé en ladite Nouvelle France le XXVe decembre mil six cens trente cinq et dont elle n'a eu nouvelle de son decedz que sur la fin du mois d'aoust dernier $1 b$
\end{abstract}

* Voir notre Revue, XVIII : 425-437.

1a Folio I, vo.

1b Le texte est en marge depuis "deceddé en" avec approbation par signatures d'Hélène Boullé et de Marie Camaret et par paraphes des notaires. 
et qui commungs estoient entre icelluy deffunt et ladite damoiselle sa vefve, trouvez et estans en la maison ou icelle damoiselle est demeu. rante, montrez et enseignez par ladite damoiselle et par Jacqueline Barbeau et Marguerite Macrelon, ses servantes, aprez serment par elles faict es mains desdits notaires de tous lesdits biens monstrer et indiquer sans aucun en receller, cacher, ne lattitter sur les peynes decrites en tel cas requises et introduictes, à elles exprimées et données à entendre par l'un desdits notaires, l'autre present, lesdits biens meubles 2 prisez et estimez par Bonnadventure Desbruyeres et Anthoine Guerin, sergent à verge jurez, priseurs, vendeurs de biens au Chatelet, ville, prevosté et vicomté de Paris qui ont serment a justice, appellez scavoir ledit Desbruyeres par ladite damoiselle de Champelain et ledit Guerin par ladite damoiselle Hersant et ce en leurs consciences, selon la juste valleur, ainsy que lesdites partyes l'ont requis au moyen de la donnation faite à ladite damoiselle de Champelain par ledit deffunt sieur son mary par leur contract de mariage passé par devant $P$. Choguillot et Arragon notaires, le XXVIIe decembre mil six cens dix, insinué au Chatelet le XIe janvier ensuivant mil six cens unze, aux sommes de deniers ${ }^{3}$ ainsy qu'il ensuict apres que ladite damoiselle Hersant a protesté que les qualitez de donnataire prise par ladicte damoiselle de Champelain ne luy puisse nuire ne prejudicier à ses droictz et actions et par ladicte damoiselle de Champelain a esté protesté au contraire et que la qualité de heritiere et creanciere prise par ladite damoiselle Hersant ne luy puisse prejudicier

Helene Boullé, Marie Camaret, Desbruyères, Guerin, Lefebvre, Duchesne Fieffé.

4 Et premierement en la cuisine de ladite maison,

Item, une paire de hastier, une pelle, une pincette et une cremelliere, prisé le tout ensemble 3 Livres

Item, en potz, platz et escuelles et autres ustancilles d'estaing commung la quantité de 77 livres, prisé la livre $10 \mathrm{solz}$, revenant le tout ensemble audit pris à la somme de $28 \mathrm{~L} 10 \mathrm{~S}$ Item, en potz, platz et escuelles et autres ustancilles d'estaing sonant a esté trouvé la quantité de vingt-trois livres, prisé la livre quinze sols, revenant le tout ensemble audit prix à la somme de $17 \mathrm{~L} 5 \mathrm{~S}$ Item, deux broches, deux lechefrittes, ung gril, deux poisles et une marmitte de fer de fonte garnie de son couvercle et cuillier, prisé le tout ensemble

Item, deux chaudrons, l'un petit et l'autre moyen avecq une cuillier et un poislon, prisé le tout ensemble

$3 \mathrm{~L}$

Item, trois chandeliers de potain et une platine prisé le tout ensemble

$3 \mathrm{~L} 10 \mathrm{~S}$

Item, un cocquemart et une marmite garnye de son couvercle de cuivre rouge, prisez ensemble

En la salle de ladite maison,

Item, une paire de chenetz de cuivre garny de leur feu prisé

Item, une table de bois de noyer assize sur son chassis, tirante par les deux boutz avecq un tapis de tapisserye à gros point a frise et a fleurs avecq une frange de laine à l'entour, prisé ensemble 5

$15 \mathrm{~L}$

2 Folio 2.

3 Le mot "deniers" est répété.

4 Folio 2, vo.

5 Folio 3. 
Item, huict chaires de bois de noyer à vertugadin couvertes de mocquettes, prisées ensemble

Item, sept plans de pareil bois et couvertz de mocquette avecq deux sieges ployans aussy couvertz de mocquette, prisées ensemble

Item, huict pieces avecq soubz bassemens et 6 entre deux de fenestre estans tant en ladite salle que en la chambre de tentures de tapisserye de Flandre à petitz personnages prisé le tout ensemble

$260 \mathrm{~L}$

Cinq tableaux peincts sur thoille garnis de leur chassis de bois ou sont despeinctz en l'un un Samson, un Saint Jerosme, une Magdeleine, une Sainte Geneviefve et une Sainte Julianne, prisez ensemble

En la deuxiesme chambre occupée par la dite veuve,

Item, une petit paire de chenetz de cuivre à olive, garnis de leur feu, prisez

$9 \mathrm{~L}$

Item, une grande table de bois de noyer assize sur son chassis avecq son tapis de mocquette, une autre petite table en ovalle ployante aussy garnye de son tapis et de mocquette, telle quelle, prisé le tout ensemble $16 \mathrm{~L}$ Item, six chaires de bois de noyer à vertugadin couvertes de velours vert, prisées ensemble

Item, quatre chaires cacquetoires couvertes de serge verte et un siege ployant couvertes de mocquette prisées ensemble 7

Item, sept tableaux de diverses grandeurs peinctz scavoir six sur thoille et un sur bois ou sont depeinctz plusieurs tableaux de devotion et une Nostre Dame, prisez ensemble

$20 \mathrm{~L}$

Item, une grande couche de bois de noyer fermant à viz, garnye de son enfonsure, une paillasse de canevas, lict et traversin de coustil, un mathelas de futaine des deux costez plain de bourre, une couverture de laine verte, un ciel, trois pentes, trois custoddes, deux bonnes-graces, le dossier, le fondz et les guenouilles, le tout de serge vert brun avecq une couverture treinante de drap reteinct, le tout garny de frange, crespine passement et molet de soye vert garny avecq trois pommes de bois doré, prisé $100 \mathrm{~L}$ En un bouge à costé de ladite chambre,

Item, une petite table de bois de chesne aveca trois chaires de bois blanc painctes, le tout tel quel, prisé ensemble

Item, un coffre de bahut rond non fermant, une petite layette de cuir à une serrure fermant à clef et une cassette de bois blanc, prisé le tout ensemble cent solz cy ...

Item, une couche bastarde fermant à viz de bois de hestre, une paillasse, un mathelas de futaine et thoille plain de bourre, un traversin de coustil plain de plume, une couverture de laine verte 8 , un tour de lict de serge vert brun contenant trois custoddes et dossier et une bonne-grasse, le tout tel quel prisé ensemble...

$15 \mathrm{~L}$

En une autre garde-robbe à costé de ladicte chambre,

Item, une table de bois de noyer assize sur son chassis prisée trois livres cy...

$3 \mathrm{~L}$

Item, une paire d'aurmoires de bois de chesne à deux guichetz fermant à clef, prisée

${ }^{6}$ Les 3 derniers mots sont rayés.

7 Fo. 3, vo.

8 Folio 4. 
Item, un coffre de bahut carré de quatre piedz de long ou environ à deux serrures fermant à clef couvert de cuir noir avecq sur deux piedz de bois blanc, prisez ensemble dix livres, cy ...

Item, un coffre fort de bois de cypres à une serrure fermant à clef, prisé six livres cy ...

$6 \mathrm{~L}$

Item, une couchette à haultz pilliers fermant à viz garnye de son enfonseure, une paillasse, un mathelas de futaine et thoile, un traversin de coustil plain de plume, une couverture de laine verte, un tour de lict de serge verte contenant trois custoddes, une bonne grasse et le dossier, prisé le tout ensemble ...

$15 \mathrm{~L}$

Item, un autre coffre-fort de bois de chesne à une serrure fermant à clef, prisé

$4 \mathrm{~L}$

Item, un autre coffre de bahut rond de quatre piedz de long ou environ à deux serrures fermant à clef prisez trois livres tournois, cy ... $\quad 3 \mathrm{~L}$ 9 Item, un petit cabinet d'Allemaigne assiz sur son pied de bois de noyer, une petite cassette couverte de cuir noir à une serrure fermant à clef et une petite aurmoire de bois de sapin, prisé le tout ensemble ... $66 \mathrm{~L}$ Item, un petit tableau peinct sur toille garny de son chassis ou est depaint une Charité, tel quel prisé vingt sols cy ... $20 \mathrm{~S}$ En un petit cabinet servant d'oratoire estant dans ladicte chambre à costé de la cheminée,

Item, un petit tapis et un orillier couvert de mocquette le tout tel quel prisé avecq deux petits morceaux de tapisserye et un rideau ... $40 \mathrm{~S}$ Item, quarente tableaux de diverses grandeurs peintz tant sur cuivre, bois, albastre que carton ou sont representez plusieurs Christ et autres personnes, prisé le tout ensemble...

$15 \mathrm{~L}$

Du samedy de relevé vingt deuxiesme jour desdicts mois et an, en continuant par lesdicts notaires à la confection du present inventaire, fut faict et inventorié ce qui ensuict Ensuivent les habitz à usaige dudict deffunt

Item, une robbe de chambre de serge de limestre coulleur de pensée, doublée de revesche avecq ung petit passement de soye et argent, prisée $\quad 15 \mathrm{~L}$ 10 Ensuivent les habitz a usage de ladite damoiselle,

Item, une juppe, une hongreline et la piece de fillatrice, le tout gris meslé, prisé le tout ensemble

Item, une robbe, une juppe et la piece de taffetas noir, prisé le tout ensemble

Item, une juppe, une hongreline de serge coulleur de Roy ou il y a ung passement d'argent à jour, une hongreline de ratine de Beauvais et les manches, prisé le tout ensemble

$12 \mathrm{~L}$

Item, une hongreline et une juppe de taffetas coullombin, une autre juppe et le corps de coste de satin mouchetté, prisé le tout ensemble $18 \mathrm{~L}$

Item, une thoilette de damas bleuf doublé de thoille, prisé $20 \mathrm{~S}$

Item, deux peaux de loultre et une peau de regnard, prisé le tout ensemble

$6 \mathrm{~L}$

Item, trois pantes de ciel et le dossier de tapisserye rehaulcée de soye à fleurs et fruictz et le fond des doz, applicque, piece et bandes de tapisserye dessus, trois custodes, deux bonnes graces et les foureaux de pilliers de taffetas changeant garny de frange crespine et molet de soye, prisé le tout ensemble cent soixante cinq livres cy

9 Folio 4, vo.

10 Folio 5. 
Item, ung tapis de table en housse de tapisserye rehaulcée de soye, doublé de bouesson bleu, prisé trente trois livres cy

Item, douze dessus de chaizes garnys de leurs dossiers, ung dessus de forme avecq le dessus de l'orillier et sept placetz, le tout de tapisserye à fleurs, les chaises garnyes avecq la forme d'une frange de soye et les sept placetz d'un frangeon et deux aultres dessus de placetz de tapisserye aussi à fleurs imparfaictz, prisé le tout ensemble soixante livres, cy $60 \mathrm{~L}$ 11 Item, deux couvertures de thoille picquée, prisée ensemble $6 \mathrm{~L}$ Item, deux carreaux couvertz de tapisserye d'un costé et de thoille de l'aultre, prisez ensemble

Ensuict le linge,

Item, six draps de thoille de chanvre neufve de deux lez chacun prisez ensemble trente livres, cy ... .

Item, six aultres draps de thoille de chanvre blanc à fleurs, prisez ensemble trente livres, cy ...

Item, deux aultres draps de thoille de chanvre à demy usez, prisez ensemble

Item, sept petitz draps de thoille de chanvre neufz de lé et demy prisez ensemble à vingt livres ...

Item, deux draps de thoille de cotton, prisez ensemble

Item, ung drap de lin et une bavette aussy de thoille de lin, prisez ensemble...

Item, deux douzaines et deux serviettes de thoille de chanvre neufves, prisées ensemble

Item, deux aultres douzaines de serviettes de thoille de chanvre prisées ensemble

Item, deux aultres douzaines de serviettes de thoille de chanvre à demyes usées, prisées ensemble

Item, six nappes de thoille de chanvre neufve de sept quartiers de long, prisé le tout ensemble...

Item, quatorze petites nappes de thoille de chanvre d'une aulne et demye de long ou environ, prisé le tout ensemble

12 Item, une nappe, une douzaine de serviette, ung buffet et deux serviettes de collation, le tout de thoille de lin ouvré, prisé le tout ensemble... $20 \mathrm{~L}$ Item, une nappe, une serviette de collation et une douzaine de serviette de thoille de lin plain, prisé le tout ensemble...

Item, une douzaine de serviettes et une nappe de thoille de lin ouvré, prisez ensemble

Item, ung petit drap de thoille de gros lin de lé et demy prisé...

$5 \mathrm{~L}$

Item, ung ciel de lassis contenant six pantes trois custodes deux bonnes graces, le dossier, le fond, la couverture de parail et les quenouilles, le tout aussy de lassis avecq une tavayolle de lassis et poinct couppé le tout, prisé ensemble trente huict livres, cy

Item, vingt deux chemises de thoille de lin à usage de femme telles queles, prisées ensemble...

Item, cinq paires de brassiéres tant de thoille que de fustaine et trois juppons aussy de thoille et fustaine prisé le tout ensemble... 
Item, ung pacquet tant de colletz, cornettes, bandeaux et autres menu linge, prisé le tout ensemble...

Ensuivent les livres...

Item, le livre des Fleurs des Sainctz, in folio couvert de veau rouge, prisé...

Item, deux livres in folio des Oeuvres de Grenade, couvertz de veau rouge, prisez ensemble... 6 L 13 Item, ung livre in-quarto relié couvert de parchemin intitullé la Triple couronne de la bienheureuse vierge, prisé $\quad 60 \mathrm{~S}$ Item, ung autre livre in quarto relié couvert de parchemin intitullé Pratique de la Perfection chrestienne, prisé

$40 \mathrm{~S}$

Item, ung autre livre in quarto relié couvert de parchemin intitulé les Epistres spirituelles de feu bienheureux François de Salle, prisé $40 \mathrm{~S}$ Item, ung livre in quarto couvert de parchemin intitullé les Méditations de Du Pont, prisé

Item, ung livre in quarto couvert de parchemin intitullé Cronicques et Instruction du pere Sainct-François, prisé le tout ensemble $40 \mathrm{~S}$ Item, ung pacquet de huict livres in octavo de divers autheurs, prisé le tout ensemble...

Item, ung livre intitullé l'Histoire des martirs du Japon, couvert de parchemin, in quarto, prisé

Item, ung autre pacquet de neuf volumes in octavo de plusieurs auteurs, prisé le tout ensemble... $60 \mathrm{~S}$ Ung autre pacquet de XIIII volumes in octavo de divers auteurs, prisé le tout ensemble...

Item, ung livre des Figures des Peres Hermites, in quarto, en taille doulce, prisé...

Item, ung aultre pacquet de dix huict volumes, in octavo et ung (de) de douze de divers auteurs, prisé le tout ensemble

Item, ung aultre pacquet de quinze volumes, in octavo de divers auteurs, prisez ensemble

14 Item, ung aultre pacquet de seize volumes, in seize, de divers auteurs, prisez ensemble...

Item, une douzaine de cousteaux a manche blanc garnys de leur gayne, prisez ensemble...

Ensuict la vaisselle d'argent,

Item, ung bassin en ovalle, deux esguieres, une salliere, ung sucrier, ung petit benoistier, six cueilliers, six fourchettes et deux flambeaux, le tout d'argent, poisson (sic) de Paris, poisant ensemble vingt-deux marcz six onces, prisé le marc vingt-cinq livres, le tout revenant ensemble audit pris... Guérin Desbruyères

568 L. 15S

Ensuivent les especes d'or et d'argent,

Item, s'est trouvé trente une livres de pieces de 20 sols 3 deniers appellez quartz d'escu, cy

Ensuivent les tiltres, papiers et enseignemens

Et a ladite damoiselle de Champlain presenté et représenté deux fueilles de grand papier dont il n'y a que deux fueilletz et la première paige du troisiesme fueillet escriptz, commanceant par ces motz 15: "Au nom du

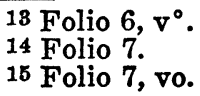


Pere, du Filz et du Sainct-esprit, moy Samuel de Champlain ..." et finissant par ces motz: "Faict le dix-septiesme de novembre à Kebec mil six cens trente cinq, en presence de ceux qui sont icy soubzsignez", signé en fin Champlain, A. de Brasdefer Chasteaufort, Derré, D. Rousseau, Bouchard, Le Tardif, Giffard, P. Gobbe, une marque allentour de laquelle est escript la marque de Guillaume Couillard, de la Ville, greffier de Quebecq, lequel escript ladicte damoiselle de Champelain dict luy avoir esté envoyé de la Nouvelle-France et luy avoir esté apporté le vendredy quatorzeiesme jour du present mois par le nommé La Treille qui disoit venir de la part du sieur Cheffault, estant ledict escript enveloppé lorsqu'elle le receust en ung pacquet avecq les commissions dudict deffunct sieur de Champelain pour le faict de son gouvernement de la Nouvelle-France, et aprez ledict pacquet ouvert, elle auroit recongneut que ledict escript est le testament et ordonnance de derniere volonté dudict deffunct sieur de Champelain son mary ayant auparavant receu nouvelles de l'envoy dudict testament par lectre missive du Pere Lallemant de la compagnie de Jesus et par le sieur Derré, à l'instant de laquelle respectivement lecture a esté faicte dudict testament par l'un des notaires soubzsignez, l'autre present, en la presence desdictes partyes comparantes, apres laquelle lecture ledict testament a esté inventorié soubz la cotte ung et demeuré es mains dudict Fieffé, l'un des notaires soubzsignez du consentement desdictes partyes ${ }^{16}$ et elles ce requerant

Boullé Camaret

affin d'estre gardé par luy pour minutte, y avoir recours et en estre delivré expedition à qui il appartiendra aux protestations respectivement faictes des partyes et mentionnées au procés verbal dudict sieur commissaire Le Vacher de la levée dudict scellé, duquel sieur commissaire ledict testament a esté paraphé en chacune paige d'icelluy ne varietur au requisitoire desdictes partyes ledict testament inventorié comme dict est ung Helene Boullé - Marie Camaret - Le Febvre - Duchesne - Fieffé Du lundy vingt-quatriesme jour desdicts mois et an en continuant par lesdicts notaires à la confection dudict inventaire fut faict et inventorié ce qui ensuict.

Et premierement, un brevet dudict Chatelet en datte du XXVIIé decembre mil six cens dix, signé Choquillot et Argon (sic) qui est le contract de mariage passé entre Maitre Nicolas Boullé, secrétaire de la chambre du Roy et Marguerite Allis, sa femme, stipulant pour Hellene Boullé, leur fille, d'une part, et ledict deffunt sieur de Champlain, d'autre, par lequel appert les futurs espoux s'estre pris aux biens et droictz à eux apartenans, qu'ilz seroient commungs entre eux selon la coustume de Paris, en faveur dudict mariage les pere et mere de ladicte future espouse auroient promis luy bailler et donner par advancement d'hoirie venant par elle à leurs successions la somme de six mil livres en deniers 17 comptans dans la veille de leurs espousailles, ledict sieur de Champlain avoir doué ladicte damoiselle de la somme de de dix-huict cens livres en douaire prefix une fois payer que le survivant d'eux deux prendroict par preciput et avant partage des biens de leur communaulté, la somme de VIc livres en meubles y declairez selon la prisée et sans ordre ou ladicte somme au choix dudict survivant, contenant aussy ledict deffunct sieur de Champlain avoir accordé que ladicte damoiselle sa future espouse jouisse sa vie durant de tous et chacuns les biens meuble et immeuble lors presens et advenir et qui pouroient apartenir audict deffunct soit par acquisition, succession, donnation que autrement pourveu qu'il n'y eust enfans dudict mariage, au

16 Folio 8.

17 Fo. 8, vo. 
dessoubz duquel brevet est la quictance passée par devant lesdicts notaires le $\mathrm{XXIX}^{\mathrm{e}}$ decembre audict an mil six cens dix de la somme de quatre mil cinq cens livres receus par ledict deffunct sieur de Champlain desdicts Boullé et sa femme sur lesdictes six mil livres et au dessoubz est l'acte de l'insinuation faicte dudict contract en datte du XI $\mathrm{XI}^{\mathbf{e}}$ janvier mil six cens unze, signé Remy et Drouart, et au dessoubz dudict acte est une coppie collationnée, signée par Anceaume et Lestoré d'une quictance passée par lesdicts sieur et damoiselle de Champlain ausdicts sieur de Boullé et sa femme de la somme de mil cinq cens livres pour le parfaict payement desdicts six mil livres. Inventorié sur ledict brevet deux Item, la procuration passée par ledict deffunct sieur de Champlain à ladicte damoiselle sa vefve en datte du XIIe febvrier mil six cens trente deux, de luy signée et de Taconnet et Groyn, notaires, inventorié sur icelle trois Item, ung escript en datte du premier juillet mil six cens trente quatre, signé Serou, qui est une promesse par luy faicte audict deffunct sieur de Champlain de la somme de cinq mil livres tournoiz pour les causes et à payer au terme y declarez, inventorié au dos de ladicte promesse quatre Item, sept pieces. La premiére est une convention imprimée du septiesme septembre 1632 au bureau de la compagnie de la Nouvelle-France, signé Lamy, par laquelle le sieur Regnault, secrétaire de la chambre 18 du Roy est comis pour recevoir de chacun des associez quatre cens livres tournoiz, au dessoubz de laquelle est la quictance dudict Regnault de ladicte somme de quatre cens livres tournoiz par luy receue dudict deffunct sieur de Champlain, l'un des associez de ladicte compagnie.

La deuxiesme autre quictance baillée et signée par ledict Regnault de la somme de cinq cens livres

La troisiesme autre quictance dudict Regnault de pareille somme, lesdictes deux quictances datées l'une comme l'autre du premier mars MVIc XXXIII faictes audict deffunct sur les trois mil livres qu'il estoit tenu pour ses contributions comme associé susdict

La quatriesme est une quictance faicte audict deffunct en datte du $17^{\mathrm{e}}$ mars audict an contenant Jehan Rozée, marchant à Rouen, avoir confessé luy avoir esté fourny par ledict deffunct la somme de IXc livres pour la contribution en la compagnie particuliére de la Nouvelle-France, ladicte partie fournie en une promesse y mentionnée

La cinquiesme est une quictance dudict Regnault du XIIe jour de novembre audict an audict deffunct de la somme de mil livres à desduire sur lesdictes contributions

La sixiesme est une quictance signée A. Cheffault du $\mathrm{XXI}^{\mathrm{e}}$ jour de juillet dernier contenant le soubzsigné commis à la requete des deniers de ladicte compagnie avoir receu dudict deffunct sieur de Champlain la somme de cinq cens livres faisant le reste desdictes trois mil livres

Et la septiesme et derniere signée $\mathrm{N}$. Liber, en datte du XXIII ${ }^{\circ}$ may 1635 , contenant ledict Liber avoir receu dudict deffunct la somme de cinq cens livres sur lesdictes trois mil livres. Inventorié lesdictes sept pieces l'une comme l'autre

cinq

Item, une quictance dattée à Quebecq le dixiesme d'aoust 1633, signé Plessis Bochart, contenant ledict Bochart avoir receu dudict deffunct le nombre de XLI escus d'or sur deux cens livres que ledict deffunct avoit receus des sieurs 19 Rozée et Cheffault sy en cas qu'on eust relasche en quelques ports et havre pour subvenir aux necessitez de ce que l'on eust

18 Fo. 9.

19 Fo. 9, vo. 
peu avoir à faire, dont il promettoit en tenir compte ausdictz Rozée et Cheffault. Inventorié sur ladicte quictance

Item, un brevet dudict Chatelet, en datte du deuxiesme mars 1620 , signée Boullé, Jacques Hersant, Marie Camaret, de Troyes et Caron, contenant Maitre Nicollas Boullé, secretaire de la Chambre du Roy, s'estre obligé envers lesdicts Hersant et sa femme de leur payer en l'acquict dudict Champlain la somme de quatre cens quarante livres au terme et pour les causes y declarez, au dos duquel brevet sont trois endossementz l'un de quarante cinq livres, ung autre de dix livres et l'autre de six vingts livres tournoiz. Inventorié sur ledict brevet En inventoriant lequel brevet ladicte damoiselle de Champlain a declaré que ce qui restoit desdictes quatre cens quarante livres a esté payé et acquité et a signé

Boulé

Item, une quictance en datte du VI ${ }^{\mathbf{e}}$ septembre 1634, signée Marie-Geneviefve de Furnes, superieure, soeur Marie Magdelaine Ivemery, assistante, soeur Magdelaine Patin, soeur Geneviefve Marestz, soeur Magdelaine-Geneviefve de Bezançon, contenant les soubzignées superieure et religieuses de la Visitation Saincte-Marie de Meaux avoir receu de ladicte damoiselle de Champlain la somme de quatre cens livres qu'elle leur avoit promis en faveur de la reception et profession de soeur Marie-Louise Sorin. Inventorié au dos de la dicte quictance huict Item, une liasse contenant vingt huict pieces aucuns non signées qui sont inventaire, compte de loiers, sentence, brevetz bail à ferme que autres, lesdictes pieces 20 cottées par premiere et derniere et paraphées par Fieffé l'un des notaires soubzsignez. Inventorié sur ladicte premiere piece pour tout Item, une autre liasse contenant cinquante cinq pieces signées et non signées qui sont quictances, sentence, actes que autres, cottées par premiere et derniere et paraphées par Fieffé, l'ung des notaires soubzsignez. Inventorié sur ladicte premiere piece pour le tout dix Item, ung recepissé de plusieurs proces signé Papin, en datte du $\mathrm{XX}^{\mathbf{e}}$ febvrier 1619 par lequel appert ledict Papin avoir receu les baillettes, contracts, donnation, adjudication et autres pieces $\mathrm{y}$ mentionnées, entre autre ung sacq de plusieurs papiers contenant la reddition du compte que la feue Le Roy a rendu à Denis Le Clerc, curateur de la dicte dame Hersant, que autres papiers des proces des deffuncts Gombault, Du Bois et Du Hamel pour les delivrer audict deffunct. Inventorié au dos dudict recepissé estant au dessoubz du memoire desdictes pieces unze Ce faict toutes les choses cy dessus inventoriez sont demourez es mains et possession de ladicte damoiselle de Champlain du consentement des autres parties par ledict proces verbal de la levée dudict scellé et ont signé

$\begin{array}{ll}\text { Helene Boullé } & \text { Le Febvre } \\ \text { Marie Camaret } & \text { Fieffé } \\ \text { Duchesne } & \end{array}$

Ensuict la teneur de la procuration dattée et mentionnée 21 en l'intitulation du present inventaire:

Par devant Pierre Teuleron, notaire tabellion royal gardenottes hereditaire en la ville et gouvernement de la Rochelle a esté present et personnellement estably le sieur Jacques Hersan, controlleur des gardes au bureau

20 Folio 10.

21 Folio 10, vo. 
des tailles foraines et domainalles de cettedicte ville de La Rochelle, y demeurant lequel de son bon gré et volonté a faict et constitué sa procuratrice generalle et specialle Marie Camaret, sa femme, laquelle il a pour l'effect des presentes et qui en despend bien et suffizament auctorizée et à icelle donne pouvoir et mandement special de pour et audict nom se transporter en la ville de Paris et autres lieux ou besoin sera pour faire recherche et perquisition des biens meubles, dhomaynes et heritages dellaissé par deffunct noble homme Samuel Champelain quand il vivoit capitaine et gouverneur pour le Roy en la Nouvelle-France, duquel la dicte Camaret est heritier presumptifves à cause de deffuncte Françoise Le Roy sa mère, à d'iceulx biens et dommaynes en venir à division et partage avecq la veufve dudict sieur Champelain ou autres coheritiers ainsy que ladicte procuratrice advisera et les biens qui escheront à sa part les vendre, arenter, afermer ou aultrement allienner, composer et tranziger les droictz successif a telles sommes, prix, termes et conditions que bon semblera à 22 ladicte Camaret et pour le tout en passer telz contractz de partage, transportz, transactions et autres actes generallement quelzconques lesquelz ledict constituant promect avoir agreable comme sy luy mesme eut esté personnellement establye à iceulx et mesme de les ratifier touteffois et quantes et en cas de reffus de venir audict partage par les autres heritiers les actionner pardevant telz juges qu'ilz appartiendra, presenter requete, sommer, protester, contester, eslire dommicille, substituer procureur, estimer, cause juger en l'ame dudict sieur constituant, poursuivre juges à jugementz deffinitif inclusivement, requerir despens, iceulx adjugees les faire taxer et liquider, affirmer, requerir et en donner acquiltz, lesquelz ledict constituans promect avoir agreable comme dessus appeller, relever, acquieser et generallement de faire en tout ce que dessus et qui en despend tout ce que feroit et faire pourroict ledict constituant sy present en personne y estoit jacoit que mandement plus special y fut requis, promectant avoir le tout pour agreable et en relever indampne sa dicte femme et procuratrice soubz l'obligation de tous ses biens et sur ce a renoncé et jugé et condampné etc... Faict à la Rochelle, en l'estude dudict notaire apres-midy, le premier jour de septembre mil six cens trente six, present Nicolas Tharay clerq et Bertrand Paillat, $m^{e}$ gainier demeurant en icelle ledict Paillat a declaré ne scavoir escrire ne signer de ce requis, signé Hersan, Tavay et Teulleron.

Ce faict ladicte procuration rendue à ladicte damoiselle Camaret $\mathbf{2 3}$.

Document qui fait suite à l'article de

ROBERT LEBLANT, 33 rue de Turin, Paris VIIIe.

22 Folio 11.

23 Archives Nationales, Minutier central, minutes de Fieffé, LXII : 138, Communication et transcription de Mme Jurgens, conservateur. 